\title{
Optimal Locating and Sizing of SSSC using Genetic Algorithm in Deregulated Power Market
}

\author{
Seyed M.H Nabavi, Student Member, IEEE \\ Kamran Khafafi, Aidin Sakhavati, Saeid Nahi \\ Department of Electrical, Tabriz Branch \\ Islamic Azad University, Tabriz, Iran
}

\begin{abstract}
The aim of this paper is presenting a genetic algorithm based method for congestion management and to maximize social welfare using one unit Static Synchronous Series Compensator (SSSC) in a double auction pool market based power systems. The aims are achieved by optimal locating and sizing one SSSC unit. In this paper, the GenCos cost functions are considered to be the quadratic form. Simulation outcomes on the modified IEEE 14 bus test system are used to show the impact of SSSC unit on the congestion management and social welfare maximization. Inclusion of the benefit functions of customer in the objective function and the utilization of a GA based algorithm for optimal locating/sizing of SSSC to guarantee fast convergence to the global optimal solution are the main contributions.
\end{abstract}

Keywords- social welfare maximization; congestion management; deregulated power market; genetic algorithms; SSSC; optimal power flow

\section{INTRODUCTION}

The restructuring in electric power industries from the last two decades was introduced with privatization in their sectors to improve their efficiency [1]. However, as the deregulation progresses among power utilities, the utility operators face new problems and challenges [2]. Furthermore, since the generation investment decision currently is in the hand of private entity, obviously there is a lack of coordination between the generation and transmission investments. Moreover, the provision of bilateral transaction, that allows GENCO and DISCO pairs to negotiate power transactions, has to lead to uncertainty in the amount and direction of power flows. Evidently, the overall consequence of this issue is the congestion in a transmission network. The issue of transmission congestion is more pronounced in deregulated and competitive markets, and it needs a special treatment [3]. In this environment, independent system operator (ISO) has to relieve the congestion, so that the system is maintained in a secure state. To relieve the congestion ISO can use mainly two types of techniques, which are as follows [4-5]:

\subsection{Cost free means:}

- Out-ageing of congested lines

- Operation of transformer taps/phase shifters

- Operation of FACTS devices, particularly series' devices

\subsection{Non-Cost free means:}

- Re-dispatching the generation amounts. By using this method, some generators back down while others increase their output. The effect of re-dispatching is that generators no longer operate at equal incremental costs.
- Curtailment of loads and the exercise of load interruption options.

Among the above two main techniques cost free means do have advantages such as not touching economical matters, so GENCO and DISCO will not be involved.

Hence, FACTS devices are utilized as one of the such technologies, which can reduce the transmission congestion and leads to better using of the existing grid infrastructure. Besides, using FACTS devices give more opportunities to ISO [1]. Various issues associated with the usage of FACTS devices are their optimal location and appropriate size, setting, cost, and modeling.

This paper deals with the optimal locating and sizing of one unit SSSC, for congestion management in competitive power markets. Up to now, the sensitivity factor methods are generally used to find the best location to enhance the static performance of the system [6]. However, there are some disadvantages for this method such that it may not capture the non-linearity associated with the power system.

Genetic algorithm as an evolutionary method can be applied as a good solution for optimization of OPF problem by incorporating FACTS devices and consequently, considering the non-linearity of the system into account. However, various optimization techniques are available to OPF problem [7].

The SSSC model is discussed in section II. Section III describes the proposed method and formulation of OPF. In Section IV, results and discussion are presented and finally. Conclusions are given in section $\mathrm{V}$.

\section{SSSC MODELING}

For static application like congestion management, FACTS devices can be accessible as Power Injection Model [1]. The injection model describes the FACTS devices as a device that injects a certain amount of active and reactive power to a node, so that the FACTS devices are presented as PQ elements.

During steady state operation, SSSC can be considered as a voltage source $V_{S e} \square \theta_{S e}$. The value of $V_{S e}^{\square} \theta_{S e}$ is adjusted according to the control scheme specified. Fig. 1(a) shows a model of a transmission line with one SSSC, which is connected between bus- $i$ and bus- $j$. The line flow change is due to using SSSC is represented as a line without voltage source with power injected at the receiving and sending ends of the line as shown in Fig. 1(b). 


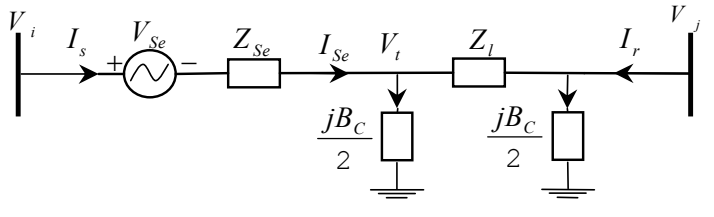

Fig. 1 (a) SSSC model

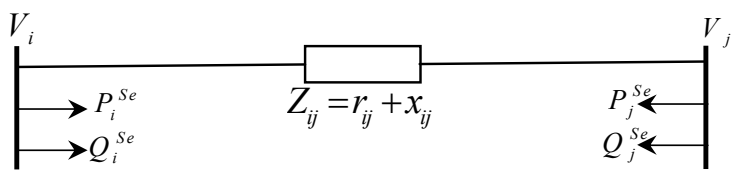

Fig.1 (b) Injection model of SSSC

The real power injections at bus- $i$ and bus- $j$ are given by:

$P_{s}^{s e}=V_{s} V_{s e}\left(1-B_{C} X_{l} / 2\right) \sin \left(\theta_{s}-\theta_{s e}\right) / H$

$P_{r}^{s e}=-V_{r} V_{s e} \sin \left(\theta_{r}-\theta_{s e}\right) / H$

Similarly, the reactive power injections at bus- $i$ and bus- $j$ can be expressed as:

$Q_{s}^{s e}=-V_{s} V_{s e}\left(1-B_{C} X_{l} / 2\right) \cos \left(\theta_{s}-\theta_{s e}\right) / H$

$Q_{r}^{s e}=V_{r} V_{s e} \cos \left(\theta_{r}-\theta_{s e}\right) / H$

where $H=X_{s e}-B_{C} X_{s e} X_{l} / 2+X_{l}$.

\section{PROPOSED METHOD}

\subsection{Genetic algorithm}

Genetic algorithm is a general optimization purpose algorithm, based on the mechanism of natural selection and genetics. It requires the evaluation function so called fitness function (FF) to assign a quality value to every solution produced. The process starts with assuming an initial random population produced and evaluated. Genetic evaluation takes place by means if three basic genetic operators:

- Parent Selection: It is done by selecting two chromosomes from the parent population based on their fitness value. The selection rule used in this paper is a simple roulette-wheel selection.

- Crossover: It is an important operator for the GA. By using this operator, two parents will get combined to form a new chromosome that inherits segments or information stored in parent chromosomes. Until now, many crossover schemes have been proposed such as single point, multipoint, or uniform. In this paper, a heuristic crossover has been used. In this kind, produced offspring is near to the parent with better fitness function as expressed by:

$O=P_{2}+R\left(P_{1}-P_{2}\right)$

$\mathrm{P} 1=$ Parent with the better fitness function

$\mathrm{R}=$ Factor of nearness to the parent - assumed 1.2

- Mutation: this operator is responsible for the injection of new information. With a small probability, random bits of the offspring chromosomes flip from 0 to 1 and vice versa and give new characteristics that do not exist in the parent's population, although in this paper it is done in real mode of each variable with using normal probability distribution for producing a random integer in the valid limit of variable. The FF evaluation and genetic evaluation take part in an iterative procedure, which ends when a maximum number of generation, are reached.

\subsection{Problem formulation}

The OPF Tools has been used normally in a pool base deregulated power markets to calculated generation dispatch and load schedules, and to manage congestion in the system. The generally accepted objective function in this environment is the maximization of social welfare. In this paper, the costs of FACTS devices are included to the social welfare maximization problem which can be expressed as:

$$
\min _{P_{G}}\left(\sum_{i=1}^{N_{G}} C_{G i}\left(P_{G}\right)-\sum_{j=1}^{N_{D}} B_{D j}\left(P_{D}\right)+\operatorname{Cost}(\text { FACTS })\right)
$$

Where $\mathrm{N}_{\mathrm{G}}$ and $\mathrm{N}_{\mathrm{D}}$ are the number of generators and loads respectively, $C_{G i}\left(P_{G}\right)$ is the bid curve of the $\mathrm{i}^{\text {th }}$ generator, and $B_{D j}\left(P_{D}\right)$ is the benefit curve for the $\mathrm{j}^{\text {th }}$ demand. subject to:

$$
\begin{array}{lr}
P_{i}(\theta, V)-P_{G i}+P_{D i}=0 & \text { for } i=1, \ldots, N_{G} \\
Q_{i}(\theta, V)-Q_{G i}+Q_{D i}=0 & \text { for } i=1, \ldots, N_{G}
\end{array}
$$

If SSSC is located in line between bus-i and bus-j, the power balance equations in bus $\mathrm{i}$ and bus $\mathrm{j}$ are given by:

$$
\begin{aligned}
& P_{i}(\theta, V)-P_{G i}+P_{D i}+P_{i}^{S e}=0 \\
& Q_{i}(\theta, V)-Q_{G i}+Q_{D i}+Q_{i}^{S e}=0 \\
& P_{j}(\theta, V)-P_{G j}+P_{D j}+P_{j}^{S e}=0 \\
& Q_{j}(\theta, V)-Q_{G j}+Q_{D j}+Q_{j}^{S e}=0
\end{aligned}
$$

Power generation limit: the limits on the maximum and minimum output of the generators are incorporated as:

$$
\begin{array}{ll}
P_{G i}^{\min } \leq P_{G i} \leq P_{G i}^{\max } & \text { for } i=1, \ldots, N_{G} \\
Q_{G i}^{\min } \leq Q_{G i} \leq Q_{G i}^{\max } & \text { for } i=1, \ldots, N_{G}
\end{array}
$$

Line flow limit: the limit on the MVA flow on a transmission line incorporated as:

$\left|S_{i j}(\theta, V)\right| \leq S_{i j}^{\max }$

Bus Voltage limit: Voltage limit at each bus is expressed as:

$V_{i}^{\min } \leq V_{i} \leq V_{i}^{\text {maz }}$

SSSC limit: the maximum and minimum values of SSSC voltage $\left(V_{S e}\right)$ and its angle $\left(\theta_{S e}\right)$ are included as:

$V_{S e}^{\min } \leq V_{S e} \leq V_{S e}^{\max }$

$\theta_{S e}^{\min } \leq \theta_{S e} \leq \theta_{S e}^{\max }$

Cost of SSSC is represented in the form of a linear equation as:

Cost $(S S S C)=S S S C \_$Capacity $\times \eta \times C R F$ 
where

$$
\begin{aligned}
& \text { Capital Recovery Factor }(C R F)=\frac{i(1+i)^{n}}{(1+i)^{n}-1} \\
& \text { Annual Capital Payment }(A C P)=P \times C R F
\end{aligned}
$$

where $\eta$ is the investment cost coefficient for per- MVA of SSSC, $i$ is a discount rate or present worth rate, $n$ is the life time of SSSC in years and $P$ is the present project cost. The capital recovery factor (CRF) indicates the equal regular payments that are equivalent to a present amount of money (Stoll (1989)). For example, $\$ 1$ today is equivalent to $\$ 0.10185$ every year for the next 20 years, assuming a discount rate (present worth rate) of $8 \%$ per annum. The annual capital payment (ACP) indicates the uniform series of annual payments (an annuity) from the beginning of the construction year through years for the useful lifetime of the project. In this paper, it is assumed that $\eta=50,000 \$$ MVA, $n=15$ years and $i=6 \%$ Per-year. Therefore, the CRF and ACP will be 0.10296 and 5148\$/MVA-year, respectively.

\subsection{Proposed algorithm}

A detailed step by step procedure for the proposed GA based social welfare maximization by incorporating all the constraint in the objective function is expressed as follows:

Step 1- Prepare a system database (generations, loads, transmission lines, cost and benefit coefficients).

Step 2- Assume GA parameters (population size (pop-size), maximum number of generators (gen-max), crossover rate (cr), and mutation rate $(\mathrm{mr})$.

Step 3- Generate chromosomes randomly, which include power generation schedule, Location and size of SSSC.

- The values of power generation corresponding to the ith generator may be expressed by the following: $P_{g i}=\lambda \times P_{g \max i} \quad 0 \leq \lambda \leq 1$

- The location of FACTS devices is described by: FACTS $_{\text {Location }}=\operatorname{INT}[$ no. lines $\times \lambda]+1, \quad 0 \leq \lambda \leq 1$ where no. lines is the number of transmission lines.

- The size of FACTS devices with respect to location of placement of the device can be described by: $\operatorname{SSSC}_{\text {Size }}=\lambda \times S S S C_{\text {Size_Max }}, 0 \leq \lambda \leq 1.0$

Step 4- Run the power flow.

Step 5- Check the equality and non-equality constraints of the system as described before.

Step 6- If any of the constraints are violated then penalty functions will be applied to each chromosome, as shown in Fig. 2(a) and 2(b).

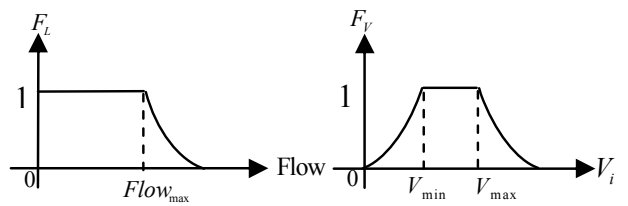

Fig. 2: Penalty functions: (a) $\mathrm{F}_{\text {lineflow_Limit }}$, (b) $\mathrm{F}_{\text {Bus_Voltage_Limit }}$

Step 7- If all the constraints are satisfied then increment pop-vn (population counter) by 1 . If pop-vn less than or equal to popsize go to step 3; otherwise go to next step.

Step 8- Calculate and store the value of objective function (maximizing social welfare or minimizing cost of generation with considering the cost of FACTS devices) corresponding to each valid generation pattern of a chromosome.
Step 9- Find and store $C_{\min }$ among all valid chromosomes and corresponding generation pattern.

Step 10- Set generation count gen-count $=1$.

Step 11- The fitness of each chromosome is defined as:

$f t_{i}=\frac{A}{\cos t_{i}} \quad, \quad i=1$ to pop - size

Where $A$ is a very large constant; Costi is the cost corresponding to the ith chromosome; $f t_{i}$ is the fitness value of function for ith chromosome.

Step 12- Construct a roulette wheel for selection process, as follows:

- Calculate the total fitness value: $F t=\sum_{i=1}^{p o p-s i z e} f t_{i}$

- Calculate the probability of selection using the formula: $p_{i}=\frac{f t_{i}}{F t} \quad i=1, \ldots$, pop - size

- Determine cumulative probabilities for each chromosome:

$q_{i}=\sum_{j=1}^{i} p_{j} \quad, \quad i=1$ to $p o p-$ size

- Generate random number $\mathrm{r}_{\mathrm{i}}$ (for $\mathrm{i}=1$, pop-size) in the range $\{0,1\}$.

- If $r_{i}<q_{i}$ then select the first chromosome; otherwise select the $m$ th chromosome such that: $q_{m-1}<r_{i}<q_{m}$

Step 13- Apply the recombination operator, crossover to the individuals in the selected population as follows:

- Generate random number $r_{i}$ (for $\mathrm{i}=1$, pop-size) in the range $\{0,1\}$

- If $r_{i}<c r$ for $\mathrm{i}=1$ to pop-size, select $i t h$ chromosome for crossover.

- If the numbers of selected chromosomes are odd, add or remove one selected chromosome randomly.

- For each pair of coupled chromosomes, generate a random integer number posi in the $[1, \mathrm{k}-1]$. The number posi indicate the position of crossing point.

Step 14- Run the power flow, then determine the generation patterns $\left(P_{g i}\right)$, voltage magnitudes and phase angles at all buses.

Also calculate power flow in each transmission line of the system.

Step 15- Check the equality and non-equality constraints of the system.

Step 16- If all the constraints in step 15 are satisfied the chromosome becomes valid otherwise it becomes invalid and hence rejected.

Step 17- Find the minimum cost among all valid chromosomes, if it is less than Cmin, store this cost in variable Cmin and also store correspondingly generation pattern.

Step 18- Add to the list of valid chromosomes, the best parents. So that total list contains the pop-size valid chromosome.

Step 19- If the mutation has been performed in the current generation; go to step 21; otherwise go to next step.

Step 20- The operator mutation is following:

- Generate a random number $r$ in the range $\{0,1\}$.

- If $r<m r$, apply mutation.

- For checking system constraints and for determining Cmin, go to step 14 . 
Step 21- If gen-count $=1$, store $C$ min in generation minimum cost Cmin-gen and also store the corresponding generation pattern.

Step 22- If gen-count $>1$ and Cmin < Cmin-gen then replace Cmin-gen by Cmin and store the corresponding generation pattern.

Step 23- Increment the generation count gen-count by 1 . If gencount < gen-max, go to step 13. Otherwise print the optimal solution among all population groups.

\section{RESULT AND DISCUSSIONS}

The proposed methodology has been implemented on IEEE 14 -bus system, which is shown in Fig. 3 [8] to demonstrate the robustness of proposed method. The network and load data for this system are taken from $[8,11]$. Line limits for IEEE 14-bus system are taken from [12]. The results obtained have been found satisfactory.

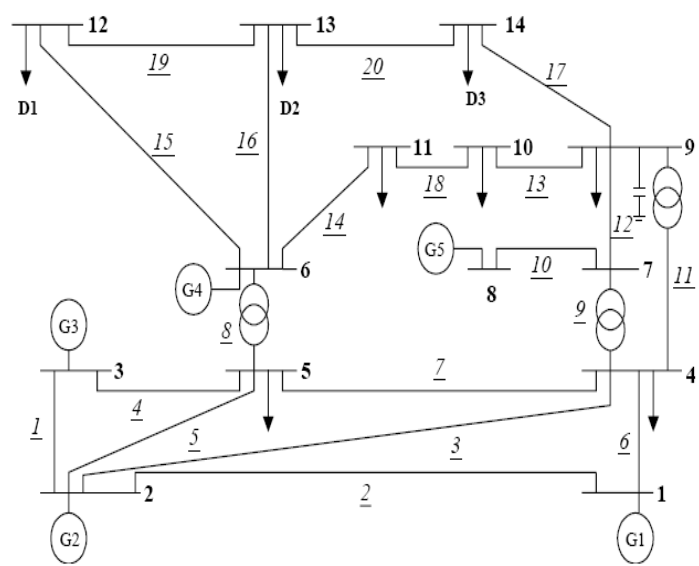

Fig. 3. IEEE 14-bus system

In this system maximum total active load is $259 \mathrm{MW}$, and maximum total generation is $772.4 \mathrm{MW}$. The results of OPF to maximize the social welfare are presented in table I, when SSSC is not placed and line limits are not considered. The optimal social welfare (minimum generation schedule) in this case is $1972.366 \$ / \mathrm{h}$. With this generation schedule, it was found that the real power flow exceeded the line flow limits in some lines and consequently, transmission congestion occurred.

As a non-cost free congestion management, the line limits are incorporated, and again optimal generation schedule was obtained. In this study social welfare is calculated $1523.926 \$ / \mathrm{h}$ (Table I). As expected, it was found that social welfare reduced after implementing the line flow limits to the optimal generation schedule.

SSSC is placed to relieve the congestion without forcing generator to operate out of their equilibrium incremental costs. The proposed algorithm is utilized to obtain the generation schedule, location and size of SSSC with objective function of maximizing social welfare. Each chromosome has 8 genes. 4 first genes correspond to generation schedule, 6th gene shows FACTS location and has an integer value in the range of $\{1,20\}$ since there are 20 lines that SSSC can be placed on, and $7^{\text {th }}$ and the $8^{\text {th }}$ correspond to SSSC voltage and angel, respectively.

Various parameters used in solving objective function using the genetic algorithm are given in table II. With implementing the GA to the objective function presented by equation 6, satisfactory results (table III.) was obtained as transmission congestion relieved and social welfare has been increased.

\section{CONCLUSIONS}

In this paper, an algorithm for congestion management based on OPF framework and using SSSC has been proposed. It is solved with the objective function of maximizing the social welfare by using GA to find optimal generation schedule, location and size of SSSC.

The proposed method was tested on IEEE 14-bus system and validated through comparison of obtained social welfare with and without SSSC. Above method only tested on IEEE 14-bus system, although it can be extended to any practical network.

\section{TABLE I. OPTIMAL GENERATION SCHEDUE WITH} AND WITHOUT LINE FLOW LIMITS CONTRAINT

\begin{tabular}{|c|c|c|c|c|c|c|}
\hline $\mathrm{B}$ & $\begin{array}{c}\mathbf{P}_{\mathrm{g}} \\
(\mathbf{M W})\end{array}$ & Total & Social & $\begin{array}{c}\mathbf{P}_{\mathbf{g}} \\
(\mathbf{M W})\end{array}$ & Total & Social \\
\hline No & without & Generation & Welfare & with line & Generation & Welfare \\
\hline & $\begin{array}{c}\text { line flow } \\
\text { limits }\end{array}$ & $\operatorname{cost}(\$ / h)$ & $(\mathbf{S} / \mathbf{h})$ & $\begin{array}{c}\text { flow } \\
\text { limits }\end{array}$ & $\operatorname{cost}(\$ / \mathbf{h})$ & $(\$ / \mathbf{h})$ \\
\hline 1 & 94.22088 & \multirow{5}{*}{1665.139} & \multirow{5}{*}{1972.366} & 97.25948 & \multirow{5}{*}{1407.154} & \multirow{5}{*}{1523.926} \\
\hline 2 & 100 & & & 100 & & \\
\hline 3 & 100 & & & \begin{tabular}{|l|}
100 \\
\end{tabular} & & \\
\hline 6 & 92.83254 & & & 48.96157 & & \\
\hline 8 & 0 & & & \begin{tabular}{|l|}
0 \\
\end{tabular} & & \\
\hline
\end{tabular}

TABLE II. GA PARAMETERS

\begin{tabular}{|c|c|}
\hline Population size & 100 \\
\hline Crossover rate & 0.9 \\
\hline Mutation rate & 0.08 \\
\hline Conversion iteration & 689 \\
\hline Maximum iteration & 1000 \\
\hline
\end{tabular}

TABLE III. OPTIMIZATION PROBLEM RESULT WITH SSSC

\begin{tabular}{|c|c|}
\hline Bus No. & Generation schedule (MW) \\
\hline 1 & 90.17146 \\
\hline 2 & 100 \\
\hline 3 & 100 \\
\hline 6 & 60.96253 \\
\hline 8 & 0 \\
\hline Total Load & 339.2016 \\
\hline Total Generation cost $(\$ / \mathrm{h})$ & 1428.592 \\
\hline SSSC location & $6-13$ \\
\hline Compensation value & $0.074 \square 37.583$ \\
\hline Social Welfare $(\$ / \mathrm{h})$ & 1660.325 \\
\hline
\end{tabular}

\section{REFERENCES}

[1] Naresh Acharya and N. Mithulananthan, "Locating series FACTS devices for congestion management in deregulated electricity markets," Electric Power Systems Research, vol. 77, pp. 352-360, March 2007.

[2] Nadarajah Mithulananthan and Naresh Acharya, "A proposal for investment recovery of FACTS devices in deregulated electricity markets," Electric Power Systems Research, vol. 77, pp. 695-703, April 2007.

[3] P. L. Jaskow and J. Tirole, "Transmission rights and market power on electric power networks," in RAND J. Econ. 31, Autumn 2000, pp. 450-487.

[4] R. S. Fang and A. K. David, "Transmission congestion management in electricity market," IEEE Transactions on Power Systems, vol. 14, No. 3, pp. 887-883, August 1999. 
[5] A. K. David, "Dispatch methodologies for open access transmission systems," IEEE Transactions on Power Systems, vol. 13, No. 1, pp. 46-53, Feb. 1998.

[6] S. N. Singh and A. K. David, "A new approach for placement of FACTS devices in open power markets," IEEE Power Engineering Review 21, pp. 58-60, Sep. 2001.

[7] Yog. Raj Sood, "Evolutionary programming based optimal power flow and its validation for deregulated power system analysis," Electric Power Systems Research, vol. 29, pp. 65-75, March 2006

[8] G. B. Shrestha and Wang Feng, "Effects of series compensation on spot price power markets," Electric Power Systems Research, vol. 27, pp. 428-436, March 2005.
[9] S.N. Singh and A. K. David, "Optimal location of FACTS devices for congestion management," Electric Power Systems Research, vol. 58, pp. 71-79, Oct. 2000.

[10] FGM. Lima, J. Munoz, I. Kockar, and FD Galiana, "Optimal location of phase shifters in a competitive market by mixed integer linear programming," $14^{\text {th }}$ PSCC, Sevilla, 24-28 June 2002.

[11] "Power system test case achieves", Retrieved 10 December 2004. From http://www.ee.washington.edu/research/pstca

[12] V. C. Ramesh and X. Li, "A Fuzzy multiobjective approach to contingency constrained OPF," IEEE Transaction on Power System, vol. 12, No. 3, pp. 1348-1354, 1997 